\title{
Actively replicating West Nile virus is resistant to cytoplasmic delivery of siRNA
}

\author{
Brian J Geiss ${ }^{1}$, Theodore C Pierson ${ }^{4}$ and Michael S Diamond*1,2,3
}

\begin{abstract}
Address: ${ }^{1}$ Departments of Medicine, Washington University School of Medicine, 660 South Euclid Avenue, Box 8051, St. Louis, MO 63110, USA, ${ }^{2}$ Molecular Microbiology, Washington University School of Medicine, 660 South Euclid Avenue, Box 8051, St. Louis, MO 63110, USA, 3 Pathology \& Immunology, Washington University School of Medicine, 660 South Euclid Avenue, Box 8051, St. Louis, MO 63110, USA and ${ }^{4}$ Department of Microbiology, University of Pennsylvania, Philadelphia, PA, 19104, USA
\end{abstract}

Email: Brian J Geiss - brian.geiss@colostate.edu; Theodore C Pierson - piersontc@niaid.nih.gov; Michael S Diamond* - diamond@borcim.wustl.edu

* Corresponding author

Published: 28 June 2005

Virology Journal 2005, 2:53 doi:10.1186/1743-422X-2-53

This article is available from: http://www.virologyj.com/content/2/I/53

(C) 2005 Geiss et al; licensee BioMed Central Ltd.

This is an Open Access article distributed under the terms of the Creative Commons Attribution License (http://creativecommons.org/licenses/by/2.0), which permits unrestricted use, distribution, and reproduction in any medium, provided the original work is properly cited.
Received: 28 May 2005

Accepted: 28 June 2005

\begin{abstract}
Background: West Nile virus is an emerging human pathogen for which specific antiviral therapy has not been developed. Recent studies have suggested that RNA interference (RNAi) has therapeutic potential as a sequence specific inhibitor of viral infection. Here, we examine the ability of exogenous small interfering RNAs (siRNAs) to block the replication of West Nile virus in human cells.

Results: WNV replication and infection was greatly reduced when siRNA were introduced by cytoplasmic-targeted transfection prior to but not after the establishment of viral replication. WNV appeared to evade rather than actively block the RNAi machinery, as sequence-specific reduction in protein expression of a heterologous transgene was still observed in WNV-infected cells. However, sequence-specific decreases in WNV RNA were observed in cells undergoing active viral replication when siRNA was transfected by an alternate method, electroporation.

Conclusion: Our results suggest that actively replicating WNV RNA may not be exposed to the cytoplasmic RNAi machinery. Thus, conventional lipid-based siRNA delivery systems may not be adequate for therapy against enveloped RNA viruses that replicate in specialized membrane compartments.
\end{abstract}

\section{Background}

West Nile virus (WNV) is a significant human and veterinary mosquito-borne pathogen that has rapidly spread across North America. Humans develop a febrile illness and a small subset progress to meningitis or encephalitis syndromes [1]. Currently, no specific therapy or vaccine has been approved for treatment or prophylaxis of WNV infection in humans.
WNV is an enveloped virus with an 11-kilobase positive strand RNA genome. It is translated directly from the genomic RNA as a single polyprotein and cleaved by cellular and viral proteases into ten mature proteins, three structural $(\mathrm{C}, \mathrm{M}$, and $\mathrm{E})$ and seven non-structural (NS1, NS2A, NS2B, NS3, NS4A, NS4B, and NS5) proteins [2,3]. Virus entry occurs by endocytosis after the E protein interacts with cellular receptor(s). Genomic viral RNA traffics to the endoplasmic reticulum (ER), where WNV protein 
translation and RNA replication occur [4]. The positive strand genomic WNV RNA that associates with the ER is competent for translation and transcription of negative strand RNA. WNV and related flaviviruses induce ER membrane proliferation and reorganization, and replicating viral RNA has been observed at these membranous structures [5-7]. Disruption of WNV protein translation and/or RNA replication blocks the viral lifecycle and aborts infection.

RNA interference (RNAi) is a cellular process that specifically degrades RNA within the cytoplasm of cells in a sequence-specific manner [8]. RNAi occurs in plants [9], nematodes [10], parasites [11,12], insects [13], and mammalian cells $[14,15]$ and is believed to function as a regulator of cellular gene expression and possibly as an innate defense against RNA viruses [16]. RNAi uses double stranded RNA (dsRNA) to target and degrade sequencespecific single-stranded RNA. The cytoplasmic ribonuclease DICER recognizes and cleaves long dsRNA molecules into 21 to 30 base pair small interfering RNA (siRNA) molecules; these associate with the RNA Induced Silencing Complex (RISC) to target and degrade complementary single-stranded RNA molecules [8].

RNAi has been used as a method to transiently disrupt various gene products to study their function [14,15,17-20]. Many mammalian viruses appear susceptible to treatment with exogenous siRNA. Cells that express virus-specific siRNA are resistant to infection by WNV [21], poliovirus $[22,23]$, influenza A $[21,24]$, HIV [25] and hepatitis C $[26,27]$ in vitro. Administration of siRNAs in vivo has modestly reduced hepatitis B antigen production $[28,29]$ and influenza A virus infection [30,31]. The sequence specific activity of siRNA against viruses has led to great interest in its potential as a new class of antiviral therapy. Nonetheless, there may be limitations with this approach as in vivo RNAi has not been demonstrated as effective as post-exposure therapy.

Previously, we demonstrated that transgenic expression of a sequence-specific siRNA prior to infection could efficiently inhibit WNV replication [21]. However, for a WNV-specific siRNA to be effective as a post-exposure therapeutic, it would need to inhibit infection in cells that are actively replicating WNV RNA. In this study, we evaluated the efficacy of siRNA against WNV that has already initiated active replication. Although cytoplasm-directed transfection of cells with siRNA prior to infection efficiently blocked WNV infection, administration after infection had little efficacy. Unlike plant viruses that encode active suppressors of RNA interference [32-34], WNV did not appear to actively inhibit the RNAi response, but rather avoided degradation by replicating in a manner that was inaccessible to the RNAi machinery.

\section{Results \\ In vitro generated siRNA inhibits WNV infection in cells}

We have previously demonstrated that plasmid expressed hairpin siRNA efficiently inhibited infection of WNV in mouse and human cell lines [21]. Because a therapeutic application of exogenously delivered rather than plasmidexpressed siRNA may be more clinically relevant, we assessed the inhibitory activity of in vitro transcribed hairpin siRNA against WNV infection. A 21-nucleotide region of the WNV capsid gene (nucleotides 312-332; Cap) was initially targeted, as this region is conserved among all WNV strains and lacks homology to known cellular genes. To demonstrate the specificity of Cap siRNA, a hairpin siRNA that targets the Influenza A M2 gene (nucleotides 18-38, M2) [21] and a mutated version of Cap siRNA (Cap Mut) that had 4 changes were also designed (Table 1). Our in vitro transcription strategy employed partially duplexed oligonucleotides containing a double stranded T7 promoter sequence (Fig 1A).

Human Huh7.5 cells were used because they were efficiently transfected with siRNA and infected with WNV. Huh-7.5 were transfected with Cap or Cap-Mut siRNA, infected with a New York strain of WNV at 18 hours after transfection, and analyzed 48 hours post-infection for levels of viral RNA by RT-PCR (Fig 1B). Pretreatment of Huh7.5 cells with Cap siRNA resulted in approximately 1 $\log$ reduction of WNV RNA, whereas pretreatment of Huh7.5 cells with Cap-Mut siRNA showed no significant reduction of WNV RNA. To confirm that RNAi also decreased WNV antigen production, siRNA-transfected Huh7.5 cells were examined for WNV envelope protein expression at 48 hours post infection. Approximately $70 \%$ of mock or TKO treated Huh-7.5 cells were positive for WNV antigen, levels comparable to that observed in cells transfected with either M2 (57\% positive) or Cap-Mut (59\% positive) siRNAs (Fig 1C). In contrast, less than 3\% of Cap siRNA transfected cells stained positive for WNV E antigen. Thus, in vitro generated sequence-specific hairpin siRNA efficiently and specifically blocked WNV RNA and antigen production in mammalian cells.

To demonstrate that siRNAs targeting different regions of the WNV genome could inhibit infection, multiple siRNAs were designed spanning the nonstructural genes of WNV (Fig 1D). Two siRNAs (5497 and 6349) targeted to the nonstructural proteins reduced WNV envelope expression by at least 4 -fold. Despite using an siRNA prediction algorithm, many of the siRNAs demonstrated little ability to inhibit envelope protein expression, possibly due to secondary structure in the WNV genomic RNA. Interestingly, treatment with combinations of siRNA did not show appreciably greater inhibition than treatment with either siRNA alone (data not shown). 
Table I: Small interfering RNA

\begin{tabular}{|c|c|c|c|}
\hline Name & Virus & Start Nucleotide & Target Sequence \\
\hline Cap & WNV Lineage I & 312 & gaacaaacaaacagcgatgaa \\
\hline Cap-Mut & WNV Lineage I & 312 & gaagaaagaaagaccgatgaa \\
\hline $\mathrm{M} 2$ & Influenza A M2 & 18 & ggtcgaaacgcctatcagaaa \\
\hline 3110 & WNV Lineage I & 3110 & gggcagttctgggtgaagt \\
\hline 3317 & WNV Lineage I & 3317 & ctacggtcaccctgagtga \\
\hline 4119 & WNV Lineage I & 4119 & gaggagcaagtctgctatgc \\
\hline 4823 & WNV Lineage I & 4823 & gtgtcaaggaggatcgact \\
\hline 5039 & WNV Lineage I & 5039 & gacggtgatgtgattgggct \\
\hline 5497 & WNV Lineage I & 5497 & gcagcaagaggttacattt \\
\hline 6337 & WNV Lineage II & 6337 & gttgaagtcatcacgaagt \\
\hline 6349 & WNV Lineage I & 6349 & gtggaagtcatcacgaagc \\
\hline 6915 & WNV Lineage I & 6915 & caacgagatgggttggcta \\
\hline 7353 & WNV Lineage I & 7353 & gaagaacgctgtagtggat \\
\hline 7693 & WNV Lineage I & 7693 & ggacgcaccttgggagaggt \\
\hline 8892 & WNV Lineage I & 8892 & ggtcaacagcaatgcagct \\
\hline 8898 & WNV Lineage I & 8898 & cagcaatgcagctttgggt \\
\hline 9095 & WNV Lineage I & 9095 & gaagcagagccatttggtt \\
\hline 9607 & WNV Lineage I & 9607 & gggaaaggacccaaagtca \\
\hline 10355 & WNV Lineage I & 10355 & gagagatatgaagacacaac \\
\hline
\end{tabular}

siRNA were generated against 19-2I nucleotide sequences corresponding to the target region of different parts of the New York 1999 WNV genome. Sequences were chosen using the SciTools RNAi design program and compared against the GenBank database to exclude sequences that may affect cellular genes.

\section{Timing of siRNA treatment affects effectiveness against WNV}

siRNA therapy in a clinical setting likely would require treatment after WNV infection has occurred. Because of this, we assessed the ability of siRNA to block WNV RNA before and after infection (Fig 2A). Huh7.5 cells were transfected with siRNAs 18 hours before infection or at 10 hours after infection. Cells were not transfected at very early times after infection because the TKO transfection reagent interfered with the ability of WNV virus to infect cells in a time-dependent manner (B. Geiss and M. Diamond, unpublished observation). By 10 hours postinfection virus had entered cells and were no longer affected by the TKO reagent. Total RNA was harvested 48 hours post-infection and analyzed for genomic WNV RNA content. As expected, pretreatment of cells with Cap siRNA, but not Cap Mut siRNA, resulted in a 10-fold reduction in WNV RNA levels. Strikingly, addition of Cap siRNA 10 hours after infection resulted in no reduction of WNV RNA. The lack of inhibitory effect of RNAi at late times after infection was not due to the emergence of resistant mutants: sequence analysis of multiple viral isolates at 48 hours post-infection from cells that had been transfected with Cap or 6349 siRNA demonstrated no mutations in the targeted viral sequences (data not shown). Thus, WNV, in contrast to poliovirus [35], did not appear to mutate to evade siRNA-triggered degradation.

\section{The rate of viral replication does not affect RNAi resistance}

The establishment of siRNA resistance could in part, be due to the ability of a rapidly replicating $\mathrm{WNV}$ to saturate the RNAi degradation machinery. To test if the replication rate affected siRNA resistance, we used an attenuated lineage II WNV that contains a GFP marker gene inserted into the 3' UTR and replicates more slowly than wild-type lineage I or II WNV [36]. Because the nucleotide sequence of the lineage II WNV was different than the lineage I WNV, a new sequence-specific siRNA was designed (6337) that targeted the analogous region on NS3 as siRNA 6349. Huh7.5 cells were transfected with Cap-Mut, 6349, or 6337 at 18 hours prior to or 10 hours after infection with the attenuated lineage II WNV, and viral RNA content was determined at 48 hours post-infection (Fig 2B). As expected, pretreatment with either Cap Mut or the lineage I-specific 6349 siRNA did not inhibit replication, whereas pretreatment with 6337 siRNA strongly blocked replication ( 60-fold). In contrast, treatment with any of the three siRNAs 10 hours after infection demonstrated no inhibitory effect. Thus, a WNV strain with a lower replication rate similarly resisted the inhibitory effects of RNAi soon after replication was established.

\section{The mode of introduction of siRNA affects the ability to establish RNAi}

Based on the timing experiments, the establishment of resistance to RNAi correlated with the onset of WNV RNA 
A

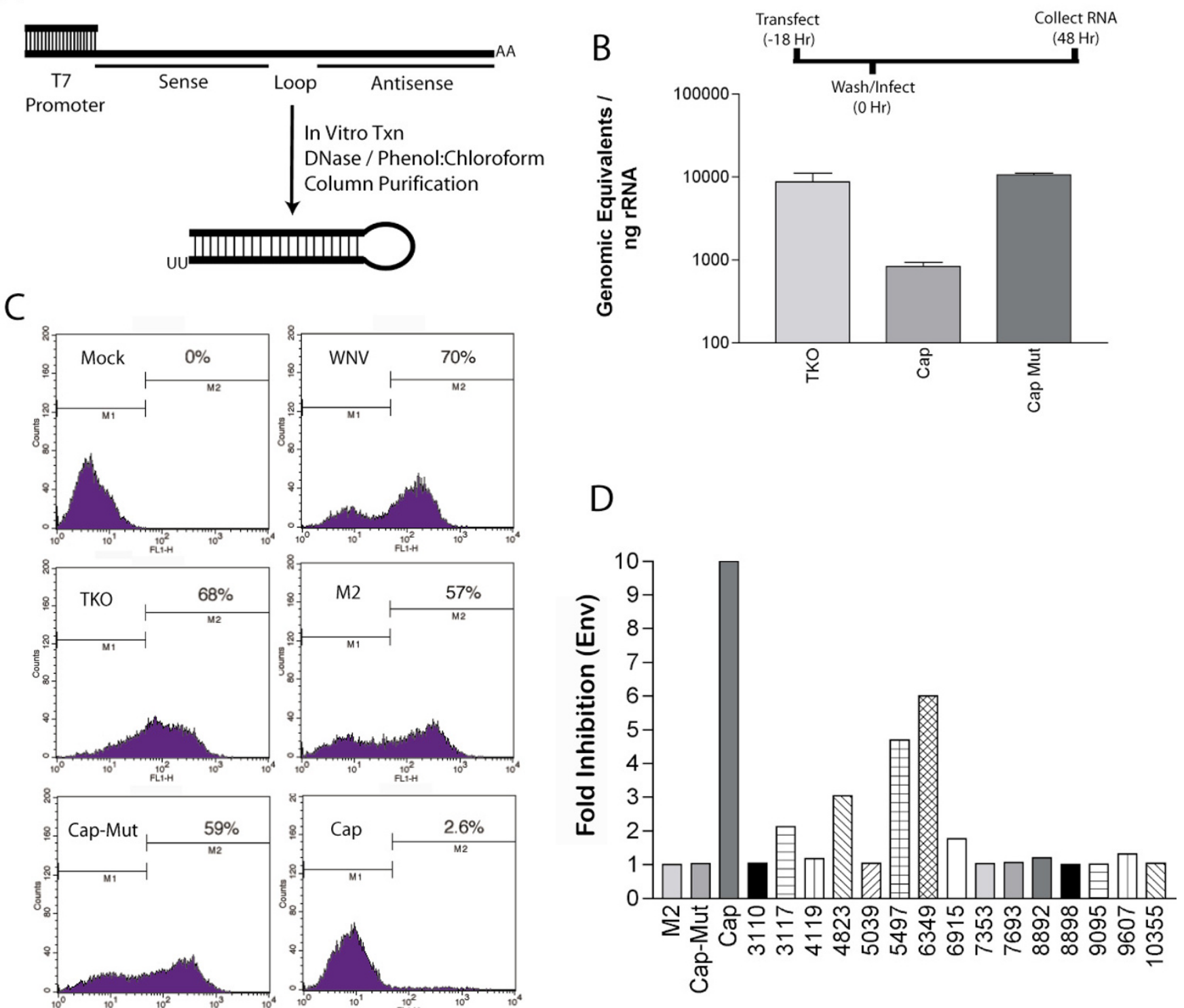

Figure I

WNV is susceptible to siRNA pretreatment. A. Scheme for generation of small hairpin siRNAs. B. Cap siRNA specifically inhibits WNV RNA accumulation. Huh7.5 cells were mock transfected or transfected with Cap siRNA or Cap Mut siRNA. Eighteen hours later cells were infected with WNV at MOI 0.I. Forty-eight hours later cells were collected and total RNA was recovered. WNV RNA was measured by quantitative real time RT-PCR. The results are an average of three independent experiments and the error bars indicate standard error of the mean. C. Capsid siRNA specifically inhibits WNV E protein expression. Huh7.5 cells were mock transfected or transfected with M2 siRNA, Cap siRNA, or Cap Mut siRNA as described above. Forty-eight hours after infection, cells were collected and processed for flow cytometry using anti-WNV envelope protein antibody EI. The results are one representative example of three independent experiments. D. Inhibitory activity of different WNV-specific siRNA. Huh7.5 cells transfected with the indicated siRNA, infected with WNV, and then analyzed for viral antigen as described in Materials and Methods. The fold inhibition was calculated after dividing the percentage of antigen positive cells from mock-transfected cells by the percentage of antigen positive cells from siRNA transfected cells. The results are one representative example of two independent experiments. 


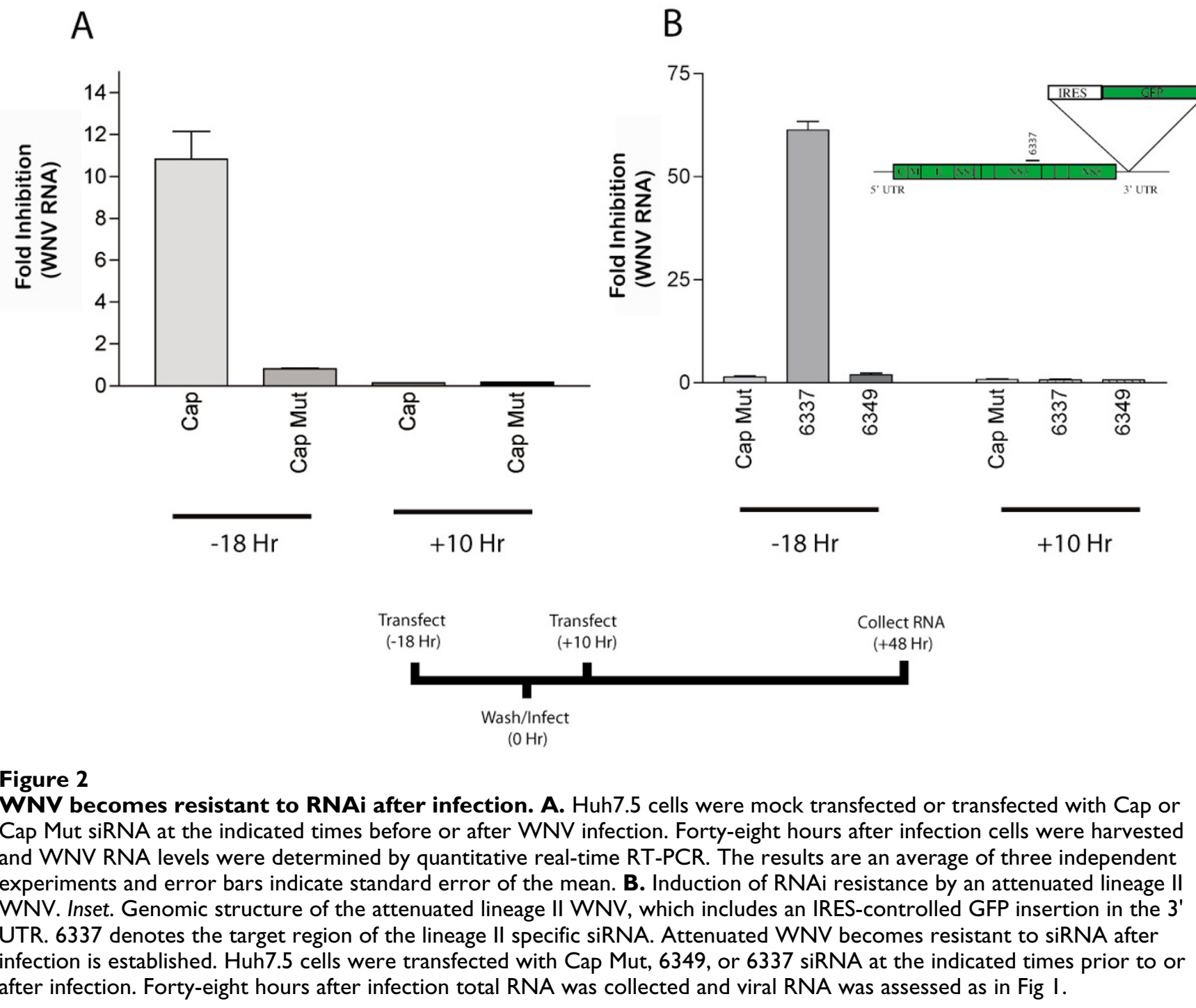

replication [37]. Shortly after infection, flaviviruses induce vesicular membrane proliferation that becomes the site of viral RNA replication $[6,7,38]$. Because the lipid-based transfection reagent targets nucleic acids to the cytoplasm (Mirus Corp, personal communication), the presence of additional membranes between the viral RNA and the cytoplasm could prevent the siRNA from reaching the actively replicating WNV RNA complex. Electroporation, in contrast, transiently opens pores in cellular membranes [39] allowing nucleic acids and cytoplasmic components to cross membranous structures such as the nucleus, endoplasmic reticulum, and potentially the membranous vesicles induced by WNV.
To determine if the mode of delivery of siRNA affected RNAi resistance, we tested whether siRNA could inhibit replication of a persistently replicating subgenomic lineage I WNV replicon; this cell line (Huh7.5-Rep) expresses the non-structural but lacks the structural proteins of WNV (Fig 3A). Huh7.5-Rep cells were transfected with 6337 and 6349 siRNAs using a lipid-based reagent or by electroporation, cultured, and assayed for reduction of NS3 antigen expression 72 hours later (Fig 3B). Repliconexpressing cells that were transfected with siRNA using the lipid-based reagent showed no significant reduction in viral protein or RNA. In contrast, using electroporation, NS3 protein levels were reduced by approximately 10 -fold 
A
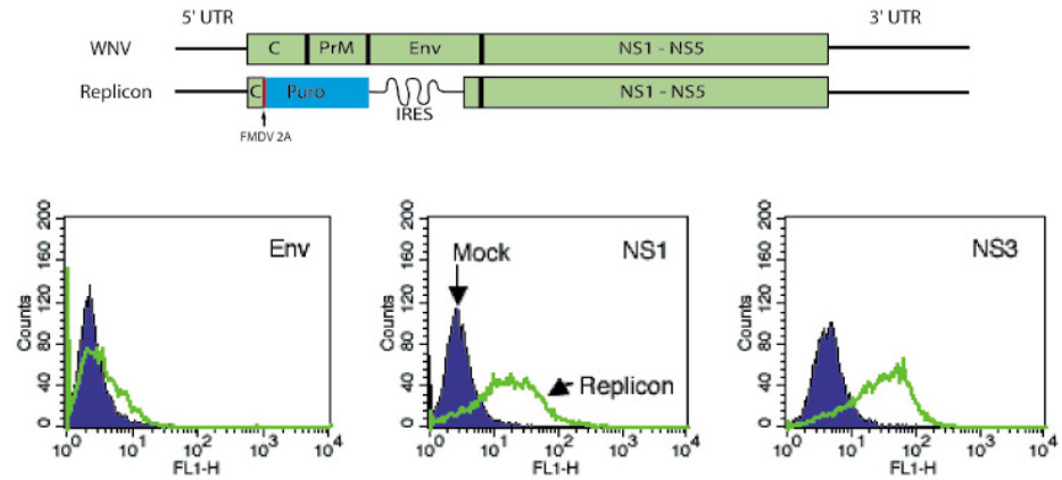

B
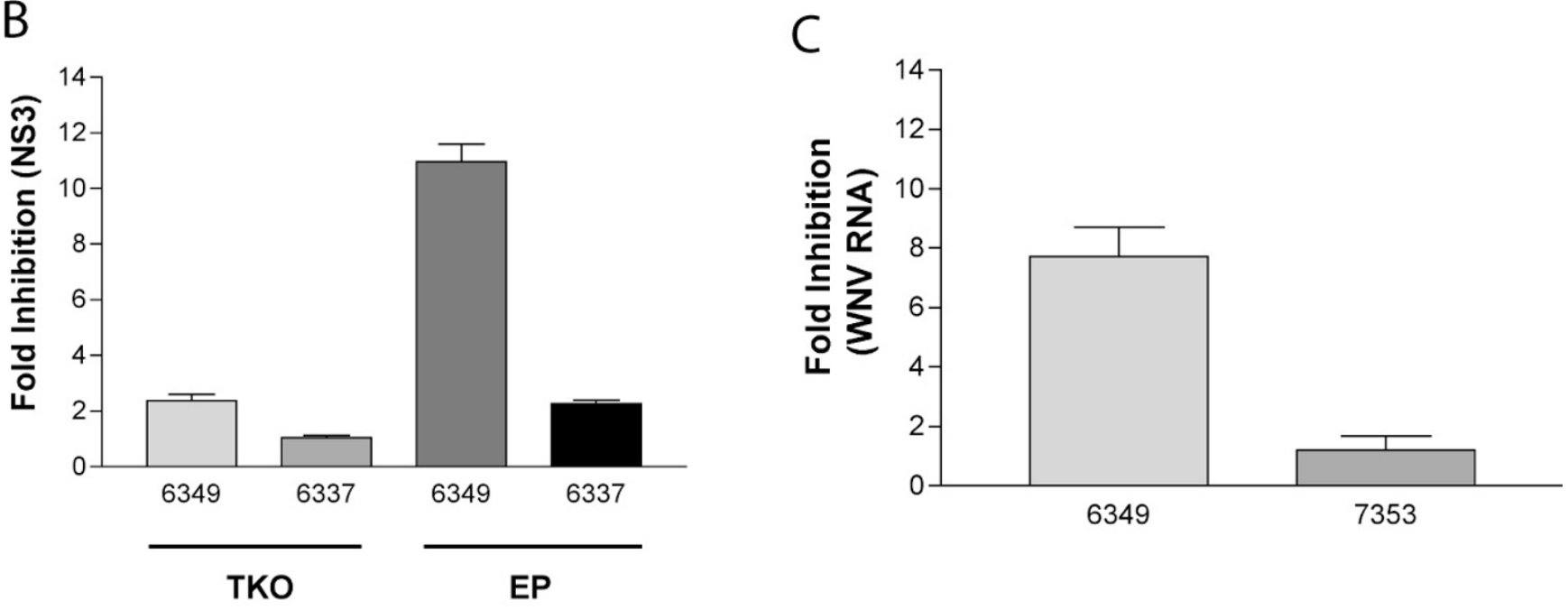

Figure 3

Mode of siRNA introduction influences WNV RNAi susceptibility. A. Huh7.5-Rep cells. (Top) Diagram of the genetic structure of the pWN5'Pur replicon. (Bottom) Flow cytometric analysis of Huh7.5 cells that express the pWN5'Pur replicon. Only non-structural proteins (e.g., NSI and NS3 but not E) are expressed. B. siRNA treatment of Huh7.5-Rep cells. Huh7.5Rep cells were mock-transfected, transfected with TKO reagent complexed with 6337 or 6349 siRNAs, or electroporated with 6337 or 6349 siRNAs. Three days later, cells were processed for viral NS3 protein expression by flow cytometry using anti-NS3 antibody (right). Fold inhibition of NS3 antigen production was determined using the formula (\% NS3 positive mock electroporated / \% NS3 positive siRNA electroporated). C. RNA analysis of Huh7.5-Rep cells electroporated with siRNA. Huh7.5-Rep cells were electroporated with 6349 or 7353 siRNA as described in Materials and Methods. Three days later, total cellular RNA was collected and viral RNA was assessed. Fold inhibition was determined by dividing the amount of viral RNA in mock electroporated samples to the amount of viral RNA in siRNA electroporated samples. The results are an average of three independent experiments and error bars indicate standard error of the mean.

by 6349 siRNA, but not by the lineage II-specific 6337 siRNA. The reduction of NS3 antigen levels correlated with $~ 7.5$-fold decreases in replicon RNA levels in the presence of 6349 (Fig 3C).

\section{Cellular localization of siRNA}

The preceding data suggested that lipid-mediated transfection delivered siRNA into the cytoplasm, whereas electro- poration at least transiently exposed replicating viral RNA to the RNAi response. However, it remained unclear whether the amount of siRNA delivered or the localization determined its ability to inhibit WNV RNA. To assess the relative amount and distribution of siRNA after transfection or electroporation, Huh7.5 cells were transfected or electroporated with Cy5-labeled Cap siRNA and analyzed 18 hours later for Cy5 expression by flow 
A

Mock

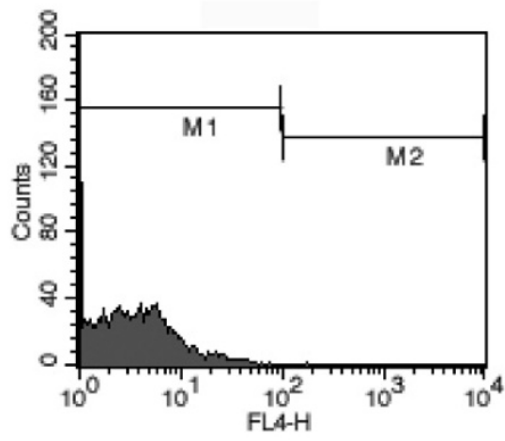

B
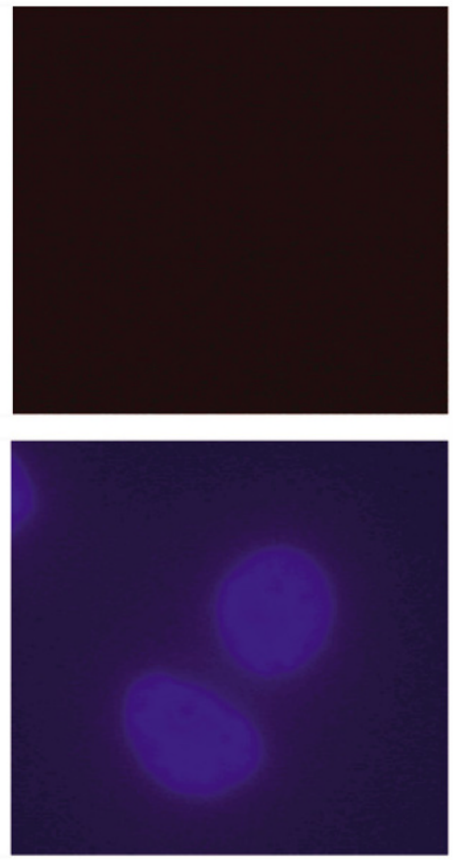

Transfected
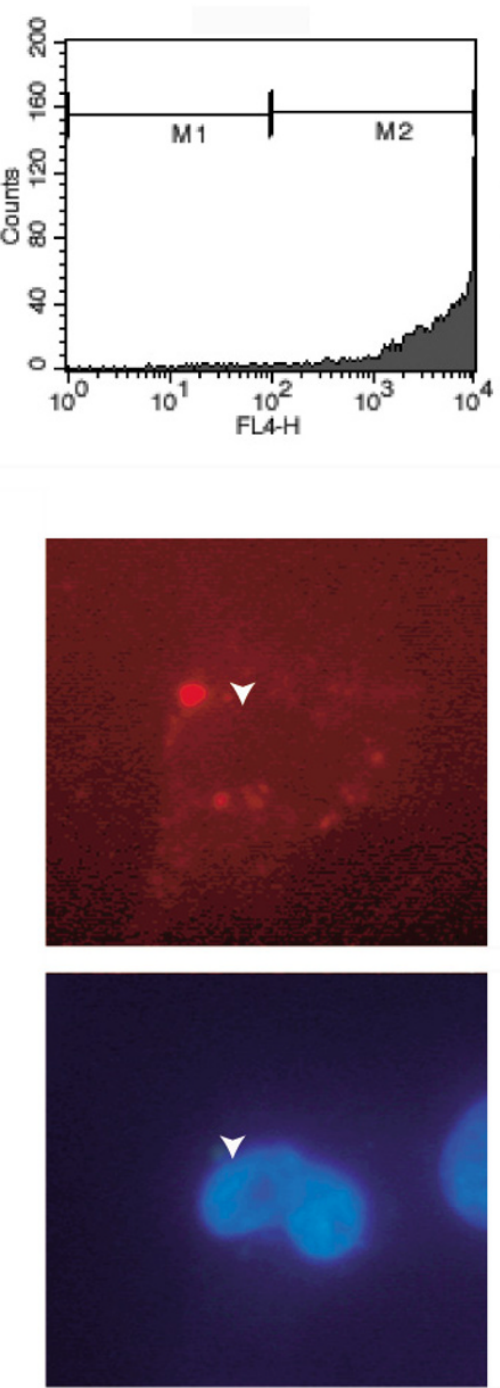

Electroporated
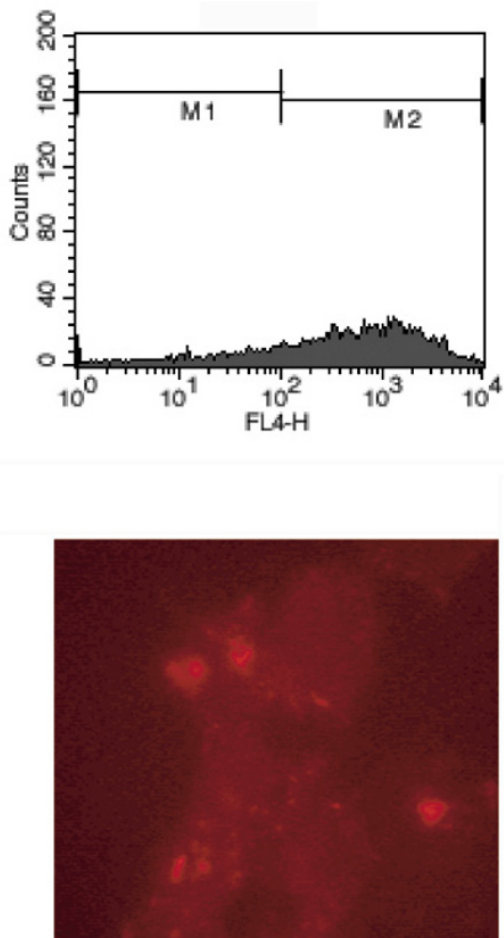

Cy5-Cap

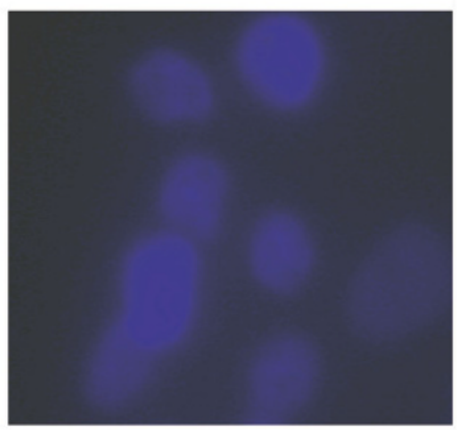

DAPI

\section{Figure 4}

Localization of siRNA. Huh7.5 cells were transfected with Cy5 labeled Cap siRNA using the lipid TKO reagent (middle panels) or by electroporation (right panels). Eighteen hours later, cells were collected and analyzed for Cy5 fluorescence by (A) flow cytometry or (B) fluorescence microscopy as described in Materials and Methods. Arrows denote the position of the nucleus. The gain in the Cy5 micrograph of electroporated cells was increased to compensate for lower levels of intracellular siRNA as compared to lipid-transfected samples.

cytometry and localization by fluorescence microscopy (Fig 4). Although all transfected cells were positive for Cy5 fluorescence, the signal was significantly higher in lipidtransfected cells than in electroporated cells (Fig 4A, geometric mean fluorescence intensity 4370 compared to 766). Microscopic analysis of lipid-transfected cells showed Cy5 signal primarily in the cytoplasm, with exclusion of Cy5 from the nucleus (Fig 4B, middle panels). In contrast, Cy5 signal was observed diffusely throughout the cell after electroporation (Fig 4B, right panels), suggesting that electroporation effectively delivered siRNA across intracellular membranes. Taken together, our data suggests that the cellular localization of siRNA appears more important than the absolute amount of siRNA delivered into the cell in determining its effectiveness against actively replicating WNV RNA. 


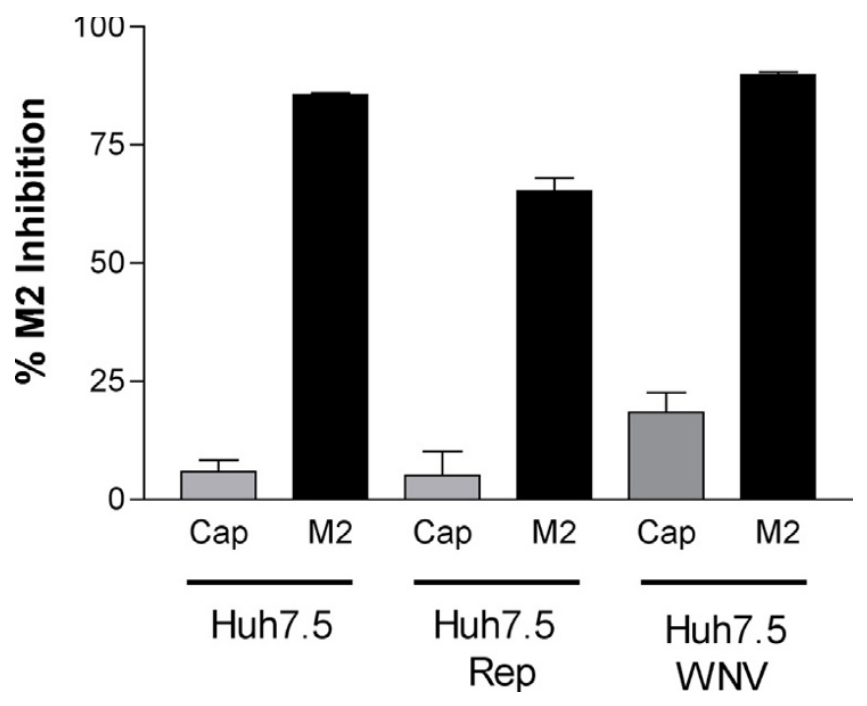

Figure 5

RNAi is active in WNV infected cells. RNAi of influenza M2 gene in cells that replicate WNV RNA. Huh7.5, Huh7.5Rep, and WNV infected Huh7.5 cells ( 8 hours post infection) were transfected with PCM2 and Cap or M2 siRNA as described in Materials and Methods. 24 hours later cells were processed by flow cytometry for $M 2$ expression using antibody I4C2. The percentage of $M 2$ inhibition was calculated according to the following formula: ( $\mathrm{I}-(\% \mathrm{M} 2$ expression of siRNA-transfected cells / \% M2 expression in cells transfected with transfection vehicle only) $\times 100$ ). The results are an average of three independent experiments and error bars indicate standard error of the mean.

RNAi against other mRNAs is intact in WNV-infected cells Although the mode and location of siRNA introduction could affect the sensitivity of actively replicating WNV to RNAi, we speculated that WNV could additionally evade RNAi by directly inhibiting one or more steps of the RNAi pathway. Targeted inhibition of the RNAi pathway has been observed in plant viruses, and has been recently reported with several mammalian viruses including LaCrosse virus, adenovirus, and influenza A virus [3234,40-43]. To determine if WNV replication directly attenuated the RNAi response, we tested the efficiency of siRNA-mediated inhibition of Influenza A virus M2 protein expression in cells that actively replicated WNV genomes (Fig 5). Mock-infected Huh7.5 cells, WNVinfected Huh7.5 cells (10 hours post-infection), and Huh7.5-Rep cells were co-transfected with an M2 expression plasmid and either Cap or M2 siRNA. Twenty-four hours later, cells were analyzed for M2 expression by flow cytometry. As expected, transfection of Cap siRNA did not significantly affect the expression of M2 in Huh7.5, Huh7.5-Rep, or WNV infected Huh7.5 cells. However, transfection of M2 siRNA effectively reduced the expres- sion of the M2 protein in all cell types, including those that actively replicated WNV RNA. Thus, WNV replication per se did not affect the establishment of RNAi of a heterologous gene.

Viral translation is necessary for WNV RNAi resistance The experiments above suggest that resistance to RNAi by WNV occurs in the setting of ongoing viral replication. During the de novo infection of a cell, translation of the input positive viral RNA strand is required before replication occurs [2]. To more finely dissect the kinetics of RNAi resistance with respect to the initiation of RNA replication, we added puromycin, a reversible inhibitor of protein chain elongation. Because puromycin inhibits cellular and viral protein translation, we first assessed how it independently affected the establishment of RNAi. Cap or Cap Mut siRNA were transfected into cells in the presence of puromycin. Four hours later, cells were infected with WNV for an additional four hours, and then free virus and puromycin were removed by serial washing of infected monolayers. Forty-eight hours after initial infection, cells were analyzed for WNV envelope protein expression (Fig 6A). When translation of full-length viral protein was reduced by puromycin, Cap siRNA more effectively inhibited WNV antigen production (90-fold versus 10 -fold reduction), suggesting that cellular translation was not necessary for priming the RNAi response and that delay of onset of WNV translation enhanced the efficiency of siRNA-mediated inhibition. Importantly, the inhibition was sequence-specific as no significant decrease in viral antigen expression was observed with the Cap Mut siRNA.

To define the kinetics of RNAi resistance around the time of initial viral replication, a puromycin time course was performed (Fig 6B). Cells were infected with WNV at MOI 0.01 and puromycin was added to Huh7.5 cells at different times $(-1,0,1,2,3,4,5,6,7$, or 8 hours) before or after infection. At 9 hours after WNV infection, all cells were transfected with Cap or Cap Mut siRNA and puromycin was removed from the medium. Cells treated with puromycin from - 1 to 1 hours post-infection were greatly protected against WNV infection. However, significant attenuation of RNAi emerged when puromycin was added just four hours after infection. These results suggest that the induction of RNAi resistance by WNV depends on translation of viral polyprotein and occurs as early as 4 hours after infection.

\section{Discussion}

In this paper we examine the ability of exogenous siRNA to inhibit the replication of WNV. Developing strategies for specific inhibition of WNV is an important goal as no current therapy exists for infected individuals. siRNA has been proposed as a potential therapy against several 

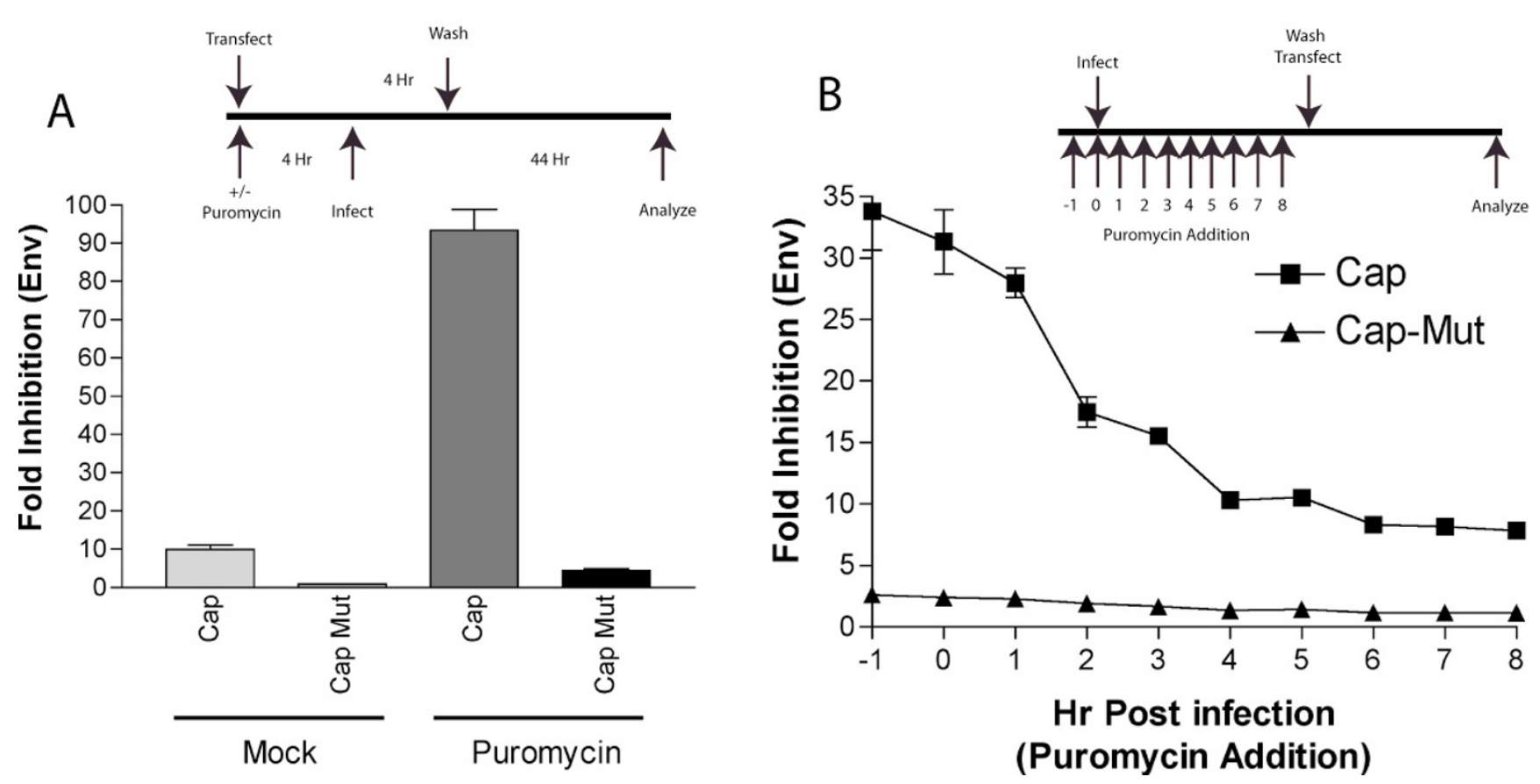

Figure 6

WNV RNAi resistance is dependent of viral translation early in infection. A. Puromycin does not interfere with RNAi. Huh7.5 cells were mock-treated or treated with $6 \mu \mathrm{g} / \mathrm{ml}$ puromycin and transfected with Cap or Cap Mut siRNA for 4 hours. Cells were washed twice, the puromycin replaced, and cells were infected with WNV at MOI 0.I. Four hours later cells were washed twice and replaced with medium that lacked puromycin. Forty-eight hours after infection, cells were collected and analyzed for WNV envelope protein expression by flow cytometry. Fold inhibition was calculated as described above. The results are an average of three independent experiments and error bars indicate standard error of the mean. B. Puromycin time course. Huh7.5 cells were infected with WNV $(\mathrm{MOI}=0.0 \mathrm{I})$, and puromycin was added at the indicated times before or after infection. At 9 hours post-infection cells were washed and transfected with Cap or Cap Mut siRNA. Forty-eight hours later, WNV envelope protein expression was assessed by flow cytometry. Fold inhibition was calculated as described in Fig $3 \mathrm{~B}$. The results are an average of three independent experiments and error bars indicate standard error of the mean.

viruses, and we have previously demonstrated that plasmid based RNAi is effective against WNV in vitro [21]. Here, we tested the ability of exogenously generated siRNA to inhibit WNV infection, as this reagent may be more practical for clinical use because there is little possibility of adverse integration into a patient's genome. Using a conventional lipid-based delivery system that targets siRNA to the cytoplasm, we confirmed that pretreatment of cells prevented infection. However, resistance to RNAi was observed when siRNA was delivered after viral translation and replication had commenced. In contrast, when siRNA was delivered by electroporation, a technique that allows macromolecules to pass across intracellular membranes, it reduced viral replication in a sequence-specific manner even if active replication was already underway. The data in this manuscript provide a first description of flavivirus resistance to RNAi during infection, and suggests a possible mechanism: WNV resists exogenously-introduced siRNA by replicating in a compartment that is sequestered behind cellular membranes.

Poliovirus is a positive strand RNA virus that replicates its genome in the cytoplasm of infected cells [44] and although susceptible to siRNA treatment, may relieve the selective pressure from siRNA by accumulating mutations in the targeted region $[22,23,35]$. In contrast, despite sequencing multiple independent isolates, we were unable to identify any mutations in siRNA-targeted regions in WNV-infected or replicon-expressing cells that were exposed to inhibitory siRNA. Also with poliovirus, some of the RNAi resistance could be overcome by administration of multiple inhibitory siRNA to disparate regions of the genome [35]. However, this was not observed with WNV, as simultaneous delivery of multiple inhibitory siRNA did not affect the resistance to RNAi in WNV- 
infected or replicon expressing cells. Thus, unlike poliovirus, WNV does not appear evade RNAi by mutating its target sequences.

WNV polyprotein translation and RNA replication within hours of infection [45]. Treatment of cells with the protein chain elongation inhibitor puromycin confirmed that establishment of RNAi resistance depended on translation of the infectious viral RNA, and that this occurred within the first four hours of infection. Electroporation of siRNA into cells expressing actively replicating WNV replicons aborted replication, suggesting both a mechanism and a means to overcome WNV-induced RNAi resistance. Nonetheless, it is possible that the method of delivery independently affects the ability of the siRNA to prime the RNAi response. The route of delivery differs between TKO transfection and electroporation (endosome versus direct transfer across membranes), and a proportion of TKO transfected siRNA may remain in endosomes for extended periods of time after transfection. However, even though five-fold more siRNA was detected in cells transfected by the TKO method, no inhibition was observed in cells that had ongoing replication of WNV RNA. We favor an alternative explanation in which WNV replication complexes are physically sequestered in a de novo generated specialized membranous compartment that is inaccessible to the cytoplasmic RNAi machinery. However, if siRNA gains access to these compartments (e.g., by electroporation) the RNAi machinery can be primed for sequence-specific degradation of viral RNA. Consistent with this, several studies have indicated that the reorganization and proliferation of endoplasmic reticulum membranes induced by flaviviruses is essential for efficient replication $[6,7,38]$. Uchil and Satchidanandam [46] proposed a model of flavivirus RNA replication in which viral dsRNA is enclosed within a double membrane structure; such a model could explain our findings. When siRNA is introduced by transfection prior to WNV infection, the cytoplasmic RNAi machinery becomes primed, and efficiently degrades infectious viral RNA after nucleocapsid penetration but before translation. In contrast, when siRNA is introduced by lipid-based transfection several hours after infection, replicating viral RNA is sequestered from the cytoplasm where the RNAi response is primed, allowing near-normal levels of replication to occur. During electroporation, however, siRNAs may be delivered across membranes and into the lumen of the viral replication compartment. How the Dicer and RISC components gain access into the replication compartment remains unknown. Although some cytoplasmic proteins may translocate across membranes during electroporation, the large size of the RNAi machinery may limit transport across membrane structures. We speculate that a small amount of Dicer and RISC gains access to the lumen of the replication compartment during its formation, and become activated when siRNA are delivered via electroporation. Clearly, additional experiments are necessary to confirm the precise mechanism.

Because treatment with siRNA in vivo would occur after an infection has been established, post-infection administration of siRNA in cell culture may reasonably predict the therapeutic utility of siRNA against individual viruses. Although many recent reports, including our own [2127], have documented that pretreatment of cells with siRNA effectively aborts infection, few studies have examined the effects of siRNA treatment on established virus infection in vitro or in vivo. For example, siRNA administration into mice 5 hours after Influenza A infection only modestly reduced viral titers [31]. Several groups have recently demonstrated that electroporation of hepatitis $\mathrm{C}$ (HCV)-specific siRNA reduced HCV RNA replication in cells expressing subgenomic replicon [26,27,47-50], results that are consistent with ours. In contrast, one study reported that siRNA transfection with oligofectamine, a lipid-based reagent, modestly reduced HCV protein expression and RNA replication in HCV-replicon expressing cells [50]. The disparity among results with lipidbased transfection systems may be reagent-based, as oligofectamine is reported to deliver a fraction of the siRNA across membranes (Invitrogen, personal communication) and thus, may transport small amounts of siRNA into the HCV replication compartment.

\section{Conclusion}

The data presented here suggests that actively replicating WNV avoids the RNAi response by replicating in a manner that is inaccessible to cytoplasm-targeted delivery of siRNA. Consistent with this, we observed little therapeutic effect of siRNA against WNV in vivo in mice (B. Geiss, M. Diamond, unpublished observation). No protection against WNV was observed when mice were treated with siRNA 24 hours after infection [51]. This lack of siRNAmediated therapeutic effect in vivo correlates with the induction of siRNA resistance that we observe in vitro. Future studies will address the role of flavivirus-induced membrane reorganization in RNAi resistance, and determine whether this mechanism is a common feature of other positive strand enveloped RNA viruses. Such information may inform the development of alternate delivery systems that allow siRNA to efficiently cross intracellular membranes and inhibit actively replicating enveloped viruses.

\section{Materials and methods Cells, viruses, and plasmids}

Baby hamster kidney cells (BHK21-15 [52]) and human Huh-7.5 hepatoma cells (gift from C. Rice, New York, NY [53]) were cultured in Dulbecco's Modified Eagle Medium with $10 \%$ fetal bovine serum as previously described [52]. 
The lineage I (3000.0259, New York 2000) and the lineage II WNV strains have been described previously [54$56]$.

The lineage I WNV subgenomic replicon plasmid pWN5'Pur was generated from a genomic clone of the New York 1999 strain (plasmids pWN-AB1 and pWNCG) provided by R. Kinney (Centers for Disease Control, Fort Collins, CO). pWN5'Pur was generated by deleting WNV nucleotides 181-2379 and fusing the first 31 amino acids of the capsid protein followed by the FMDV 2A autocleavage peptide [57] and the puromycin $\mathrm{N}$-acetyl transferase (PAC) gene [58]. The EMCV IRES [53] was placed downstream of the PAC stop codon, so that translation of the WNV structural proteins begins at nucleotide 2380 (Methionine 794). The lineage II WNV genomic clone containing a Not I restriction site or an IRES-driven GFP have been described $[36,56]$.

DNA template for replicon RNA transcription was prepared by linearization of pWN5'Pur with Xba I restriction endonuclease followed by phenol:chloroform extraction and ethanol precipitation. Replicon RNA was generated using the Amplicap T7 High Yield Message Maker kit (Epicenter Technologies, Madison WI). T7 RNA transcripts were electroporated into Huh7.5 cells as described below to generate Huh7.5-Rep cells. Huh7.5-Rep cells were stably selected with $5 \mu \mathrm{g} / \mathrm{ml}$ puromycin (Sigma-Aldrich, St. Louis MO). Reverse transcriptase PCR was performed as previously described $[59,60]$ using primers $3026 \mathrm{~F}$ (5'TGACTCGAAGATCATTGGAA) and 4496R (5'ATCCATATCTTCCAAGGTGC). Plasmid pCAGGS M2 was described previously [21].

\section{siRNA production, RNA and DNA transfection}

siRNA were generated in vitro by run-off transcription from a partially double-stranded oligonucleotide template. Oligonucleotides that contained the T7 RNA polymerase promoter (5'AAATTTAATACGACTCACTATA) were annealed to a 75-mer oligonucleotide, which contained an antisense T7 RNA polymerase promoter sequence, 19-21 nucleotides corresponding to the target sequence (Table 1), a 10 nucleotide loop region, 19-21 nucleotides complementary to the target sequence, and two adenine residues (5'AA (sense 21) AACCAGAAGA (antisense 21) TATAGTGAGTCGTATTAAATTT). Targeted sequences were chosen using the SciTools RNAi design program (Integrated DNA Technology, Coralville, IA) and compared against the GenBank database to exclude sequences that may affect cellular genes. Polyacrylamide gel electrophoresis (PAGE)-purified oligonucleotides were purchased from Integrated DNA Technologies (Coralville, IA).
siRNA were transcribed using the MegaShortScript T7 Transcription Kit (Ambion, Austin, TX) according to the manufacturers recommendations with the exception that 200 additional units of T7 RNA Polymerase (Ambion) were included in each $20 \mu \mathrm{l}$ reaction. RNA transcription reactions were carried out at $37^{\circ} \mathrm{C}$ for 1.5 hours, treated with DNAse I for $15 \mathrm{~min}$, extracted with phenol and chloroform, desalted over ChromaSpin TE-10 columns (BD Biosciences, Palo Alto, CA), and stored at $-80^{\circ} \mathrm{C}$. siRNA were quantified by PAGE gel electrophoresis and UV spectroscopy. Cy5 labeled siRNA was generated by adding 1 mM Cy5-UTP (Amersham Biosciences, Piscataway, NJ) to transcription reactions. Huh7.5 cells were transfected at various times before or after infection with $1 \mu \mathrm{g}$ siRNA using $5 \mu \mathrm{l}$ Trans-IT TKO reagent (Mirus Corp., Madison, $\mathrm{WI}$ ) according to the manufacturer's instructions. Electroporations were performed using a BTX ElectroSquarePorator as described [53]. In all electroporation experiments, 5 $\times 10^{6}$ cells were electroporated in the presence of $50 \mu \mathrm{g}$ siRNA. TKO transfected cells were washed twice with fresh DMEM media before infection with WNV. Viral antigen expression in WNV-infected cells was analyzed by flow cytometry (FACSCalibur, Becton-Dickinson) using the anti-WNV envelope E1 monoclonal antibody [61]. Monoclonal antibodies against NS1, (9NS1; K. Chung and M. Diamond, unpublished data) and NS3, (clone E1E6; R. Beatty and E. Harris, unpublished data) were used to detect viral antigen in cells that expressed WNV replicons. Goat-anti-mouse IgG -FITC (Sigma-Aldrich, St. Louis MO) was used to detect primary antibodies. Plasmid DNA transfections were performed using Trans-IT LT1 reagent (Mirus Corp., Madison WI) at a ratio of $8 \mu \mathrm{l}$ transfection reagent / $1 \mu \mathrm{g}$ plasmid DNA according to the manufacturers recommendation. For experiments involving co-transfection of plasmid and siRNA, plasmid DNA and siRNA were separately complexed with the appropriate transfection reagent, mixed together and incubated at room temperature for 15 minutes, and added to cells.

\section{Quantitative real-time reverse transcriptase PCR}

WNV infected samples were collected at 48 hours postinfection, and total RNA was isolated using RNEasy RNA extraction columns (Qiagen, Valencia, CA) according to the manufacturer's instructions. Real-time reverse transcriptase PCR and quantitation of WNV transcripts was performed as previously described [62]. Quantitation of Lineage I replicon RNA was performed using a primerprobe set directed towards the 3' UTR WNV genome (forward primer 5'AGAGTGCAGTCTGCGATAGTGC; probe 5' Fam ACAAAGGCAAACCAACGCCCCA TAMRA; reverse primer 5'CCTTTCGCCCTGGTTAACA). Quantitation of Lineage II genome was performed using a primer set directed towards the 3' UTR of the Lineage II WNV genome (forward primer 


\section{ACAAAGGCAAAACATCGCCCCA TAMRA; reverse primer 5'CCСТTCTCCCTGGTTAACA).}

\section{Fluorescence microscopy}

Cy5-Cap transfected or electroporated Huh7.5 cells were plated onto Lab-Tek glass slides (Nalge Nunc, Naperville IL) and incubated for 18 hours at $37^{\circ} \mathrm{C}$. Cells were fixed in cold $4 \%$ paraformaldehyde, washed extensively, permeabilized with $0.5 \%$ Triton X-100, and mounted in Prolong Gold Plus DAPI mounting reagent (Molecular Probes, Eugene OR). Slides were visualized and digitally captured using a Zeiss Axioskop microscope (Zeiss Microimaging, Thornwood, NY).

\section{Competing interests}

The author(s) declare that they have no competing interests.

\section{Authors' contributions}

BJG designed and constructed the subgenomic WNV replicons, designed and performed all experiments, and helped draft the manuscript. TCP designed, constructed, and tested the WNV-GFP clone and critically reviewed the manuscript. MSD and BJG designed the study, and MSD helped draft and critically review the manuscript.

\section{Acknowledgements}

We thank Richard Kinney for the WNV plasmids $p W N-A B I$ and $p W N$ CG, Andy Pekosz and Matt McCown for the plasmid pCAGGS M2 and for monoclonal antibody $14 \mathrm{C} 2$, and Robert Beatty and Eva Harris for monoclonal antibody EIE6. We would also like to thank Elizabeth Moulton for helping to set up the siRNA production system, Keril Blight for use of the electroporation apparatus, Andy Pekosz and Robert Doms for critical review of this manuscript and helpful discussions, and the Blight, Olivo, Leib, Pekosz, Diamond, Klein, and Morrison laboratories for helpful discussions. This work was supported by a grant from the NIH (I U0I Al53870). BG was supported by a Ruth L. Kirschstein National Research Service Award, 5 T32 Al07/72-25,

\section{References}

I. Petersen LR, Marfin AA: West Nile virus: a primer for the clinician. Ann Intern Med 2002, 137:173-179.

2. Lindenbach: Flaviviridae: The Viruses and Their Replication. In Fields Virology Volume 1. 4th edition. Philadelphia, Lippincott, Williams, and Wilkins; 200I:99I-I04I.

3. Chambers TJ, McCourt DW, Rice CM: Production of yellow fever virus proteins in infected cells: identification of discrete polyprotein species and analysis of cleavage kinetics using regionspecific polyclonal antisera. Virology 1990, 177:159-174.

4. Mackenzie JM, Jones MK, Young PR: Immunolocalization of the dengue virus nonstructural glycoprotein NSI suggests a role in viral RNA replication. Virology 1996, 220:232-240.

5. Mackenzie JM, Westaway EG: Assembly and maturation of the flavivirus Kunjin virus appear to occur in the rough endoplasmic reticulum and along the secretory pathway, respectively. J Virol 200I, 75:10787-10799.

6. Westaway EG, Khromykh AA, Mackenzie JM: Nascent flavivirus RNA colocalized in situ with double-stranded RNA in stable replication complexes. Virology 1999, 258:108-117.

7. Westaway EG, Mackenzie JM, Kenney MT, Jones MK, Khromykh AA: Ultrastructure of Kunjin virus-infected cells: colocalization of NSI and NS3 with double-stranded RNA, and of NS2B with NS3, in virus-induced membrane structures. J Virol I997, 7I:6650-666I.

8. Meister G, Landthaler M, Dorsett $Y$, Tuschl T: Sequence-specific inhibition of microRNA- and siRNA-induced RNA silencing. Rna 2004, 10:544-550.

9. Que Q, Jorgensen RA: Homology-based control of gene expression patterns in transgenic petunia flowers. Dev Genet 1998, 22:100-109.

10. Fire A, Xu S, Montgomery MK, Kostas SA, Driver SE, Mello CC: Potent and specific genetic interference by double-stranded RNA in Caenorhabditis elegans. Nature 1998, 391:806-81 I.

II. Ngo H, Tschudi C, Gull K, Ullu E: Double-stranded RNA induces mRNA degradation in Trypanosoma brucei. Proc Natl Acad Sci U S A 1998, 95: | 4687-14692.

12. Ullu E, Tschudi C, Chakraborty T: RNA interference in protozoan parasites. Cell Microbiol 2004, 6:509-5I9.

13. Hoa NT, Keene KM, Olson KE, Zheng L: Characterization of RNA interference in an Anopheles gambiae cell line. Insect Biochem Mol Biol 2003, 33:949-957.

14. Paddison PJ, Caudy AA, Bernstein E, Hannon G], Conklin DS: Short hairpin RNAs (shRNAs) induce sequence-specific silencing in mammalian cells. Genes Dev 2002, 16:948-958.

15. Paddison PJ, Caudy AA, Hannon G]: Stable suppression of gene expression by RNAi in mammalian cells. Proc Natl Acad Sci U S A 2002, 99: | $443-1448$.

16. Waterhouse PM, Wang MB, Lough T: Gene silencing as an adaptive defence against viruses. Nature 200 I, 4I I:834-842.

17. Elbashir SM, Lendeckel W, Tuschl T: RNA interference is mediated by 2I- and 22-nucleotide RNAs. Genes Dev 200I, I5:188-200

18. Elbashir SM, Harborth J, Lendeckel W, Yalcin A, Weber K, Tuschl T: Duplexes of 21 -nucleotide RNAs mediate RNA interference in cultured mammalian cells. Nature 200I, 4I I:494-498.

19. Garrus JE, von Schwedler UK, Pornillos OW, Morham SG, Zavitz KH Wang HE, Wettstein DA, Stray KM, Cote M, Rich RL, Myszka DG, Sundquist WI: TsgIOI and the vacuolar protein sorting pathway are essential for HIV-I budding. Cell 200I, 107:55-65

20. Sui G, Soohoo C, Affar el B, Gay F, Shi Y, Forrester WC: A DNA vector-based RNAi technology to suppress gene expression in mammalian cells. Proc Natl Acad Sci U S A 2002, 99:55 I 5-5520.

21. McCown M, Diamond MS, Pekosz A: The utility of siRNA transcripts produced by RNA polymerase $i$ in down regulating viral gene expression and replication of negative- and positive-strand RNA viruses. Virology 2003, 3 | 3:5 14-524.

22. Gitlin L, Karelsky S, Andino R: Short interfering RNA confers intracellular antiviral immunity in human cells. Nature 2002, 41 8:430-434.

23. Saleh MC, Van Rij RP, Andino R: RNA silencing in viral infections: insights from poliovirus. Virus Res 2004, 102:1 I-17.

24. Ge Q, McManus MT, Nguyen T, Shen CH, Sharp PA, Eisen HN, Chen $J$ : RNA interference of influenza virus production by directly targeting mRNA for degradation and indirectly inhibiting all viral RNA transcription. Proc Natl Acad Sci U S A 2003, 100:27|8-2723.

25. Jacque JM, Triques K, Stevenson M: Modulation of HIV-I replication by RNA interference. Nature 2002, 4I 8:435-438.

26. Wilson JA, Jayasena S, Khvorova A, Sabatinos S, Rodrigue-Gervais IG, Arya S, Sarangi F, Harris-Brandts M, Beaulieu S, Richardson CD: RNA interference blocks gene expression and RNA synthesis from hepatitis $C$ replicons propagated in human liver cells. Proc Natl Acad Sci U S A 2003, 100:2783-2788.

27. Randall G, Grakoui A, Rice CM: Clearance of replicating hepatitis $C$ virus replicon RNAs in cell culture by small interfering RNAs. Proc Natl Acad Sci U S A 2003, 100:235-240.

28. McCaffrey AP, Nakai H, Pandey K, Huang Z, Salazar FH, Xu H, Wieland SF, Marion PL, Kay MA: Inhibition of hepatitis B virus in mice by RNA interference. Nat Biotechnol 2003, $21: 639-644$.

29. Giladi H, Ketzinel-Gilad M, Rivkin L, Felig Y, Nussbaum O, Galun E: Small interfering RNA inhibits hepatitis $B$ virus replication in mice. Mol Ther 2003, 8:769-776.

30. Tompkins SM, Lo CY, Tumpey TM, Epstein SL: Protection against lethal influenza virus challenge by RNA interference in vivo. Proc Natl Acad Sci U S A 2004, I 0 I:8682-8686.

31. Ge Q, Filip L, Bai A, Nguyen T, Eisen HN, Chen J: Inhibition of influenza virus production in virus-infected mice by RNA interference. Proc Natl Acad Sci U S A 2004, I0 I:8676-868I. 
32. Chen J, Li WX, Xie D, Peng JR, Ding SW: Viral virulence protein suppresses RNA silencing-mediated defense but upregulates the role of microrna in host gene expression. Plant Cell 2004, 16:1302-1313.

33. Kasschau KD, Xie Z, Allen E, Llave C, Chapman EJ, Krizan KA, Carrington JC: PI/HC-Pro, a viral suppressor of RNA silencing interferes with Arabidopsis development and miRNA unction. Dev Cell 2003, 4:205-217.

34. Qu F, Ren T, Morris T): The coat protein of turnip crinkle virus suppresses posttranscriptional gene silencing at an early initiation step. J Virol 2003, 77:5 I I-522.

35. Gitlin L, Stone JK, Andino R: Poliovirus Escape from RNA Interference: Short Interfering RNA-Target Recognition and Implications for Therapeutic Approaches. J Virol 2005, 79: 1027-1035.

36. Pierson TC, Diamond MS, Ahmed AA, Valentine LE, Davis CW, Samuel MA, Hanna SL, Puffer BA, Doms RW: An infectious West Nile virus that expresses a GFP reporter gene. Virology 2005, 334:28-40

37. Takegami T, Hotta S: Synthesis and localization of Japanese encephalitis virus RNAs in the infected cells. Microbiol Immunol 1990, 34:849-857.

38. Mackenzie JM, Jones MK, Westaway EG: Markers for trans-Golgi membranes and the intermediate compartment localize to induced membranes with distinct replication functions in flavivirus-infected cells. J Virol 1999, 73:9555-9567.

39. Weaver JC: Electroporation: a general phenomenon for manipulating cells and tissues. / Cell Biochem 1993, 5 I:426-435.

40. Joost Haasnoot PC, Cupac D, Berkhout B: Inhibition of virus replication by RNA interference. J Biomed Sci 2003, 10:607-616.

41. Li WX, Li H, Lu R, Li F, Dus M, Atkinson P, Brydon EW, Johnson KL, Garcia-Sastre A, Ball LA, Palese P, Ding SW: Interferon antagonist proteins of influenza and vaccinia viruses are suppressors of RNA silencing. Proc Natl Acad Sci U S A 2004, I0 I: I 350- I 355

42. Lu S, Cullen BR: Adenovirus VAI noncoding RNA can inhibit small interfering RNA and MicroRNA biogenesis. J Virol 2004, 78: $12868-12876$

43. Soldan SS, Plassmeyer ML, Matukonis MK, Gonzalez-Scarano F: La Crosse Virus Nonstructural Protein NSs Counteracts the Effects of Short Interfering RNA. J Virol 2005, 79:234-244.

44. Baltimore D, Eggers HJ, Franklin RM, Tamm I: Poliovirus-induced RNA polymerase and the effects of virus-specific inhibitors on its production. Proc Natl Acad Sci U S A 1963, 49:843-849.

45. Lindenbach $\mathrm{BD}$, Rice $\mathrm{CM}$ : trans-Complementation of yellow fever virus NSI reveals a role in early RNA replication. I Virol 1997, 71:9608-9617.

46. Uchil PD, Satchidanandam V: Architecture of the flaviviral replication complex. Protease, nuclease, and detergents reveal encasement within double-layered membrane compartments. J Biol Chem 2003, 278:24388-24398.

47. Sen A, Steele R, Ghosh AK, Basu A, Ray R, Ray RB: Inhibition of hepatitis $C$ virus protein expression by RNA interference. Virus Res 2003, 96:27-35.

48. Zhang J, Yamada O, Sakamoto T, Yoshida H, Iwai T, Matsushita Y, Shimamura $\mathrm{H}$, Araki H, Shimotohno K: Down-regulation of viral replication by adenoviral-mediated expression of siRNA against cellular cofactors for hepatitis C virus. Virology 2004 320:135-143.

49. Kronke J, Kittler R, Buchholz F, Windisch MP, Pietschmann T, Bartenschlager R, Frese M: Alternative approaches for efficient inhibition of hepatitis $C$ virus RNA replication by small interfering RNAs. J Virol 2004, 78:3436-3446.

50. Takigawa $Y$, Nagano-Fujii M, Deng L, Hidajat R, Tanaka M, Mizuta $H$, Hotta $H$ : Suppression of hepatitis $C$ virus replicon by RNA interference directed against the NS3 and NS5B regions of the viral genome. Microbiol Immunol 2004, 48:59I-598.

5I. Bai F, Wang T, Pal U, Bao F, Gould LH, Fikrig E: Use of RNA interference to prevent lethal murine west nile virus infection. Infect Dis 2005, 191:। | 48-1 I54.

52. Diamond MS, Roberts TG, Edgil D, Lu B, Ernst J, Harris E: Modulation of Dengue virus infection in human cells by alpha, beta, and gamma interferons. J Virol 2000, 74:4957-4966.

53. Blight KJ, Kolykhalov AA, Rice CM: Efficient initiation of HCV RNA replication in cell culture. Science 2000, 290:1972-1974.

54. Ebel GD, Dupuis AP, Ngo K, Nicholas D, Kauffman E, Jones SA, Young D, Maffei J, Shi PY, Bernard K, Kramer LD: Partial genetic charac- terization of West Nile virus strains, New York State, 2000. Emerg Infect Dis 200I, 7:650-653

55. Wengler G, Gross HJ: Studies on virus-specific nucleic acids synthesized in vertebrate and mosquito cells infected with flaviviruses. Virology 1978, 89:423-437.

56. Yamshchikov V, Mishin V, Cominelli F: A new strategy in design of +RNA virus infectious clones enabling their stable propagation in E. coli. Virology 200I, 28I:272-280.

57. de Felipe P: Skipping the co-expression problem: the new 2A "CHYSEL" technology. Genet Vaccines Ther 2004, 2: I 3.

58. Vara J, Malpartida F, Hopwood DA, Jimenez A: Cloning and expression of a puromycin $\mathrm{N}$-acetyl transferase gene from Streptomyces alboniger in Streptomyces lividans and Escherichia coli. Gene 1985, 33:197-206.

59. Diamond MS, Shrestha B, Marri A, Mahan D, Engle M: B cells and antibody play critical roles in the immediate defense of disseminated infection by West Nile encephalitis virus. J Virol 2003, 77:2578-2586.

60. Lanciotti RS, Kerst AJ, Nasci RS, Godsey MS, Mitchell CJ, Savage HM, Komar N, Panella NA, Allen BC, Volpe KE, Davis BS, Roehrig JT: Rapid detection of west nile virus from human clinical specimens, field-collected mosquitoes, and avian samples by a TaqMan reverse transcriptase-PCR assay. J Clin Microbiol 2000, 38:4066-407I.

6I. Oliphant T, Engle M, Nybakken GE, Doane C, Johnson S, Huang L, Gorlatov S, Mehlhop E, Marri A, Chung KM, Ebel GD, Kramer LD, Fremont DH, Diamond MS: Development of a humanized monoclonal antibody with therapeutic potential against West Nile virus. Nat Med 2005, I I:522-530.

62. Diamond MS, Shrestha B, Mehlhop E, Sitati E, Engle M: Innate and adaptive immune responses determine protection against disseminated infection by West Nile encephalitis virus. Viral Immunol 2003, 16:259-278.

Publish with Biomed Central and every scientist can read your work free of charge

"BioMed Central will be the most significant development for disseminating the results of biomedical research in our lifetime. "

Sir Paul Nurse, Cancer Research UK

Your research papers will be:

- available free of charge to the entire biomedical community

- peer reviewed and published immediately upon acceptance

- cited in PubMed and archived on PubMed Central

- yours - you keep the copyright

Submit your manuscript here:

http://www.biomedcentral.com/info/publishing_adv.asp
BioMedcentral 